\title{
Tendência e diferenciais socioeconômicos da mortalidade por câncer de colo de útero no Estado do Paraná (Brasil), 1980-2000
}

\author{
Socioeconomic trends and differentials in mortality \\ due to cervical cancer in the State of Paraná (Brazil), 1980-2000
}

Erildo Vicente Müller ${ }^{1}$

Maria Gabriela Haye Biazevic ${ }^{2}$

José Leopoldo Ferreira Antunes ${ }^{2}$

Edgard Michel Crosato ${ }^{2}$

${ }^{1}$ Universidade Estadual de Ponta Grossa. Av. General Carlos Cavalcanti 4748, Uvaranas. 84030-900 Ponta Grossa PR. evicmu@hotmail.com 2 Departamento de Odontologia Social, Faculdade de Odontologia, Universidade de São Paulo.

\begin{abstract}
The scope of this paper is to discuss the evolution of mortality due to cervical cancer in the State of Paraná, Brazil, between 1980 and 2000 and analyze the socioeconomic differentials in each region of the State. Mortality data were gathered from the System for Information on Mortality by age and town of residence. Age-adjusted death rates were calculated for 22 regions of the state in each year. Comparative analysis evaluated socioeconomic indicators associated with regions that showed either stationary or increasing mortality trends. Cervical cancer deaths increased in the state of Paraná, with an annual percentage increase of $1.68 \%$ (1.20 to 2.17, 95\% confidence interval). Most of the regions presented a stationary trend of cervical cancer deaths. The comparison of regions presenting an increasing trend indicated poorer socioeconomic indices for the former set: regions with an increase in cervical cancer mortality had a significantly higher illiteracy rate $(p<0.001)$, percentage of individuals older than 15 years with less than 4 years schooling ( $p=0.001$ ), and lower per-capita income $(p=0.025)$ and $h u$ man development index ( $p=0.023)$. An increasing mortality trend was thus observed throughout the State and areas with higher mortality rates had poorer socioeconomic indices.
\end{abstract}

Key words Cervical cancer, Epidemiology, Mortality, Cancer, Statistics models, Trends
Resumo O objetivo deste artigo é discutir a evolução da mortalidade por câncer de colo de útero no Estado do Paraná entre 1980 e 2000 e analisar seus diferenciais socioeconômicos em cada região. Taxas de mortalidade ajustadas por idade foram calculadas para as 22 regionais de saúde do Estado a cada ano. Análises comparativas avaliaram indicadores socioeconômicos associados com regiões que apresentaram tendência estacionária e crescente de mortalidade. A mortalidade por câncer de colo uterino cresceu no Estado como um todo a uma taxa de 1,68\% (IC 1,20-2,17) ao ano. A maior parte das regiões apresentou tendência estacionária de mortalidade por câncer de colo de útero. As regionais com tendência de aumento na mortalidade apresentaram proporção significativamente mais elevada de analfabetismo $(p<0,001)$ e de adultos (15 anos ou mais) com menos de 4 anos de estudo ( $p=0,001)$, e renda per capita $(p=0,025)$ e IDH $(p=0,023)$ inferiores. Houve tendência de aumento na mortalidade em todo o Estado; as regiões que contribuíram para o aumento experimentaram piores indicadores socioeconômicos.

Palavras-chave Câncer de colo de útero, Epidemiologia, Mortalidade, Câncer, Modelos estatísticos, Tendências 
Introdução

O câncer de colo do útero (CA de colo de útero) é o segundo mais comum entre mulheres no mundo e a maior causa de morbidade e mortalidade $^{1}$, com cerca de 470 mil casos novos em todo o mundo ${ }^{2-4}$. Quase $80 \%$ desses casos ocorrem em países em desenvolvimento ${ }^{2,3,5}$ e em populações com pior condição socioeconômica ${ }^{4,6}$.

No Brasil, o CA de colo de útero ocupa o quinto lugar em mortalidade entre todas as neoplasias malignas, com mais de 20.000 casos por ano, ficando em segundo lugar com relação às neoplasias do sexo feminino ${ }^{7}$.

No município de São Paulo (SP), entre 1980 e $1990^{8}$, a tendência da mortalidade por CA de colo de útero, ajustada pela idade, manteve-se estável (em torno de 5,2/100.000) entre 1980 e 1999; as taxas relativas ao corpo de útero experimentaram aumento, e sem especificação de localização registrou movimento descendente. Em Salvador (BA), houve um decréscimo de 50,6\% no coeficiente padronizado de mortalidade por CA de colo de útero entre 1979 e 1997, e os coeficientes de mortalidade variaram de 17,6/100.000 em 1979 a 8,7/100.000 em 1997. No Rio Grande do Sul, observou-se aumento anual na mortalidade de 0,17 no período de 1979 a $1998^{10}$; o coeficiente anual médio foi de 7,58/100.000 mulheres ${ }^{10}$.

Tem-se observado redução da incidência e da mortalidade em países desenvolvidos ${ }^{11}$, em decorrência de programas sistemáticos de rastreamento das populações de risco ${ }^{12}$. Falhas nesses programas têm sido consideradas como o fator mais importante associado a elevados coeficientes da doença ${ }^{3}$. O programa Viva Mulher, que foi implantado em 1997 pelo Ministério da Saúde, fornece dados sobre os programas de rastreamento de CA de colo de útero no Brasil e cita a baixa cobertura do teste de Papanicolau, que cobre entre $8 \%$ e $10 \%$ da população feminina em torno de 20 anos de idade ${ }^{13}$.

O monitoramento de informações epidemiológicas sobre CA de colo de útero é necessário para o planejamento de estratégias adequadas de detecção precoce e redução da carga de doença na população. O presente estudo objetivou descrever a evolução da mortalidade por CA de colo de útero no Paraná entre 1980 e 2000, e avaliar sua associação com condições socioeconômicas das regiões do Estado.

\section{Métodos}

Trata-se de estudo descritivo, utilizando o método epidemiológico com delineamento de série temporal (estudo de tendências), de base territorial, para levantamento da mortalidade por CA de colo de útero no Estado do Paraná, entre os anos de 1980 e 2000. A variável dependente foi a mortalidade por CA de colo de útero indicada como causa básica nos atestados de óbito. Foram coletadas informações sobre mortalidade através do banco de dados de óbitos do Sistema de Informações sobre Mortalidade (SIM) ${ }^{14}$ do Ministério da Saúde, de 1980 a 2000, código 180 da Classificação Internacional das Doenças, 9a revisão (CID 9) ${ }^{15}$ até 1995 , e código C53 da 10a revisão, a partir de $1996^{16}$.

A população de estudo incluiu todos os óbitos de mulheres com idade igual ou superior a 15 anos, residentes no Estado do Paraná no período de 1980 a 2000, classificados segundo CA de colo de útero no SIM. As faixas etárias para caracterização dos grupos foram as definidas pelo SIM: 15-19; 20-29; 30-39; 40-49; 50-59; 60-69; 70$79 ; 80$ anos ou mais. As informações sobre as regionais foram obtidas através de dados da Secretaria Estadual de Saúde do Estado do Paraná $(\text { SESA-PR })^{17}$, por meio do Departamento de Planejamento e Controle.

Foram descritos os coeficientes de mortalidade por CA de colo de útero para o Paraná como um todo, padronizados por faixa etária em relação à população feminina do Brasil, conforme informada pelos censos de 1980, 1991 e 2000. Para os anos intercensitários, foram empregadas estimativas populacionais fornecidas pelo Instituto Brasileiro de Geografia e Estatística (IBGE) ${ }^{18}$.

As taxas padronizadas foram calculadas pelo método direto ${ }^{19}$ segundo ano e faixa etária para as 22 regionais de saúde do Estado: Paranaguá, Metropolitana, Ponta Grossa, Irati, Guarapuava, União da Vitória, Pato Branco, Francisco Beltrão, Foz do Iguaçu, Cascavel, Campo Mourão, Umuarama, Apucarana, Londrina, Cornélio Procópio, Jacarezinho, Toledo, Cianorte, $\mathrm{Pa}$ ranavaí, Maringá, Telêmaco Borba e Ivaiporã. Para a caracterização das tendências temporais, foi utilizado o procedimento de Cochrane-Orcutt $^{20}$ para análise de autorregressão em séries temporais, e o cálculo da taxa anual de incremento de mortalidade.

Os indicadores de condição socioeconômica das regionais de saúde do Estado do Paraná foram calculados através das informações obtidas do censo realizado em $2000^{18}$ : renda familiar, co- 
eficiente de desemprego, coeficiente de Gini, coeficiente de analfabetismo e indicadores de nível educacional. A correlação entre mortalidade e fatores sociodemográficos foram verificados através do teste de correlação de Pearson. As análises estatísticas foram realizadas utilizando-se o programa estatístico Stata, adotando-se nível de significância de $5 \%$.

\section{Resultados}

O Estado do Paraná apresentou um total de 4.006 óbitos por CA de colo de útero de 1980 a 2000, com aumento da taxa de mortalidade no período estudado (Gráfico 1). A taxa de crescimento anual foi de 1,67\% (IC 1,05-2,29) no Estado do Paraná como um todo (Tabela 1), com apenas três regionais contribuindo para a tendência estadual.

De fato, a maioria das regiões do Estado do Paraná apresentou tendência estável, com exceção de Campo Mourão, com taxa de crescimento anual de 4,91\% (IC 0,83-9,16), Guarapuava, de $8,18 \%$ (IC 4,89-11,57) e Ponta Grossa, com incremento anual de 5,50\% (IC 0,14-11,15) nos óbitos por CA de colo de útero.
As regionais do Estado do Paraná que apresentaram tendência de aumento na mortalidade apresentaram também piores taxas em alguns indicadores socioeconômicos, tais como maior taxa de analfabetismo $(\mathrm{p}<0,001)$, maior proporção de moradores com menos de quatro anos de estudo $(\mathrm{p}=0,001)$, renda per capita $(\mathrm{p}=0,025)$ e

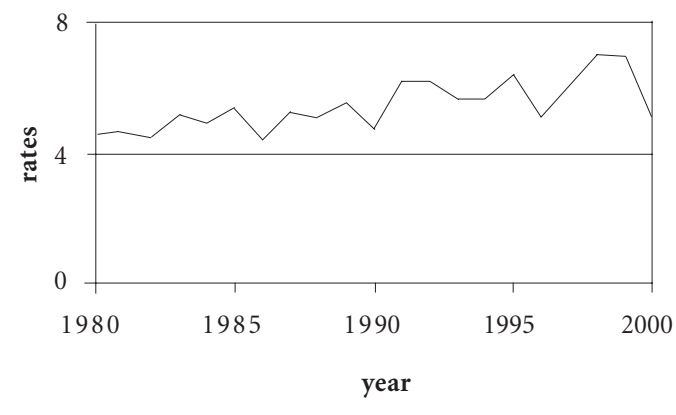

Gráfico 1. Mortalidade por câncer de colo de útero, coeficientes padronizados por grupo etário, por 100.000 habitantes - Estado do Paraná, 19802000.

Tabela 1. Taxa de crescimento anual dos coeficientes padronizados de mortalidade por câncer de colo de útero segundo Regional de Saúde, Estado do Paraná, 1980-2000 - Estado do Paraná, 2004.

\begin{tabular}{lcrrl}
\hline \multicolumn{1}{c}{ Regional de Saúde } & Taxa de crescimento anual & IC (95\%) & Tendência \\
\hline Apucarana & 3,08 & -0.64 & 6,94 & estacionária \\
Campo Mourão & 4,91 & 0,83 & 9,16 & crescente \\
Cascavel & 1,70 & $-1,59$ & 5,09 & estacionária \\
Cianorte & $-2,08$ & $-6,33$ & 2,36 & estacionária \\
Cornélio Procópio & 4,27 & $-0,23$ & 8,97 & estacionária \\
Foz do Iguaçu & 5,20 & $-0,63$ & 11,37 & estacionária \\
Francisco Beltrão & 1,39 & $-1,10$ & 3,95 & estacionária \\
Guarapuava & 8,18 & 4,89 & 11,57 & crescente \\
Irati & 0,00 & $-3,87$ & 4,02 & estacionária \\
Ivaiporã & $-1,34$ & $-4,65$ & 2,08 & estacionária \\
Jacarezinho & 3,49 & $-1,79$ & 9,05 & estacionária \\
Londrina & 0,1 & $-0,84$ & 1,87 & estacionária \\
Maringá & 1,40 & $-2,35$ & 5,31 & estacionária \\
Metropolitana & 0,74 & $-0,57$ & 2,07 & estacionária \\
Paranaguá & $-0,33$ & $-3,61$ & 3,06 & estacionária \\
Paranavaí & $-0,57$ & $-4,00$ & 2,97 & estacionária \\
Pato Branco & $-0,05$ & $-3,60$ & 3,63 & estacionária \\
Ponta Grossa & 5,50 & 0,14 & 11,15 & crescente \\
Telêmaco Borba & $-1,60$ & $-7,35$ & 4,51 & estacionária \\
Toledo & 2,99 & $-0,63$ & 6,75 & estacionária \\
Umuarama & 2,37 & $-1,39$ & 6,27 & estacionária \\
União da Vitória & 1,05 & $-1,86$ & 4,05 & estacionária \\
Total & 1,67 & 1,05 & 2,29 & crescente \\
& & & &
\end{tabular}


índice de desenvolvimento humano (IDH) $(\mathrm{p}=0,023)$ inferiores às regionais que apresentaram tendência estável. A Tabela 2 mostra as características socioeconômicas das regionais com tendência crescente e decrescente de mortalidade.

\section{Discussão}

Em todo o mundo, tem-se observado redução na incidência e mortalidade por câncer de colo de útero no século passado, e esse comportamento vem sendo atribuído ao maior acúmulo de conhecimentos sobre a sua etiologia, além da implementação de programas de educação em saúde e orientação para o comportamento sexual ${ }^{21}$. Porém, os países em desenvolvimento teriam se beneficiado em menor proporção do que os mais desenvolvidos, e na América Latina as taxas de incidência e mortalidade não vêm apresentando amplas reduções conforme esperado ${ }^{22}$.

Em São Paulo, a mortalidade por câncer de colo de útero tem-se apresentado estacionária ${ }^{23}$, ou seja, sem amplas modificações em um período de vinte anos. Outro estudo observou declínio na mortalidade, na cidade de São Paulo, apenas nos distritos possuidores de melhor condição socioeconômica ${ }^{24}$.

No Estado do Paraná, o presente estudo observou comportamento semelhante a São Paulo, com tendência global estacionária e alguns distritos, justamente os possuidores de indicadores socioeconômicos mais pobres, com tendência crescente na mortalidade. Os dados de mortalidade por câncer no Estado do Paraná constituem fonte adequada para análise de mortalidade, já que foram avaliados como possuidores de boa qualidade no período analisado ${ }^{25}$. Porém, a classificação indicativa dos óbitos por CA de colo de útero não leva em consideração os óbitos classificados como câncer cervical, sem realização de correções na mortalidade ${ }^{26}$.

Dentre os fatores associados que aumentam a suscetibilidade ao CA de colo de útero estão incluídas precárias condições de vida relacionadas diretamente a condições socioeconômicas precárias $^{6,27}$. Falta de acesso aos serviços de saúde também está relacionada a essa problemática, especialmente à presença de programas de rastreamento populacional ${ }^{28}$.

De acordo com a Organização Mundial da Saúde (OMS), para que haja um impacto epidemiológico na diminuição das taxas de incidência e mortalidade pelo CA de colo de útero, a cobertura dos programas de rastreamento deve ser de $85 \%$ das mulheres ${ }^{29}$. No Brasil, a cobertura de tais programas foi descrita como sendo precária ${ }^{30}$.

Os valores médios de coberturas anuais e trienais de citologias do colo uterino divulgados pelo Programa de Prevenção e Controle do Câncer Ginecológico no Estado do Paraná entre 1998 e 2004 foram, respectivamente, de $23,17 \%$ e $68,84 \%$ na faixa etária de 25 a 59 anos $^{17}$. As taxas de cobertura para as regionais de saúde que apresentaram tendência crescente de mortalidade, no ano de 2004, foram: 66,3\% para Campo Mourão, 39,3\% para Guarapuava e 39\% para Ponta

Tabela 2. Comparação da mortalidade por câncer de colo de útero entre regionais com tendência estacionária e com tendência crescente - Estado do Paraná, 2004.

\begin{tabular}{lccr}
\hline \multicolumn{1}{c}{ Características socioeconômicas } & $\begin{array}{c}\text { Regionais com } \\
\text { tendência estacionária }\end{array}$ & $\begin{array}{c}\text { Regionais com } \\
\text { tendência crescente }\end{array}$ & p \\
\hline \% população rural & 24,065 & 29,969 & 0,385 \\
Taxa de analfabetismo & 20,979 & 24,170 & $<0,001$ \\
\% terminaram 2 ${ }^{\circ}$ grau & 14,325 & 11,907 & 0,331 \\
Anos de estudo (média) & 4,148 & 3,729 & 0,123 \\
\% domicílios com água encanada & 78,684 & 74,388 & 0,518 \\
\% domićlios com esgoto & 27,316 & 22,037 & 0,567 \\
\% domićlís com coleta de lixo & 76,783 & 71,356 & 0,400 \\
\% menos de 4 anos de estudo aos 15 anos & 33,243 & 38,076 & 0,001 \\
Renda per capita & 207,432 & 176,053 & 0,025 \\
Coeficiente de Gini & 0,553 & 0,573 & 0,278 \\
Coeficiente de Theil & 0,527 & 0,562 & 0,370 \\
Índice de Desenvolvimento Humano (IDH) & 0,7429 & 0,718 & 0,023 \\
\hline
\end{tabular}


Grossa ${ }^{17}$. Nas regionais que apresentaram tendência de estabilidade, a proporção de mulheres cobertas foi, em geral, superior a $70 \%{ }^{17}$.

As limitações das estatísticas vitais estão presentes nos estudos de mortalidade. Os sub-registros de mortes devidas ao câncer podem ser resultado da não exatidão da definição da causa de morte, e menores taxas de mortalidade pela doença podem ser resultados de classificação desses óbitos como causas mal definidas ou não especificadas nos registros de mortes. Antunes e Wünsch-Filho ${ }^{31}$ identificaram essa problemática para o caso de CA de colo de útero.

Deve-se, ademais, levar em consideração as limitações do estudo ecológico, que faz a análise dos dados em um âmbito coletivo que não permite inferir os resultados em nível individual ${ }^{19}$.

\section{Considerações finais}

Os resultados obtidos na presente pesquisa levam-nos a concluir que programas de rastreamento sistematizados devem ser repensados e desenvolvidos objetivando atingir diretamente os municípios como estratégia para a diminuição da incidência e mortalidade por câncer do colo de útero.

Campanhas educacionais devem ser realizadas visando conscientizar a população da importância do exame de Papanicolaou, priorizando os grupos populacionais que se encontram na faixa etária de maior risco e que nunca realizaram o exame, assim como aqueles cujas condições socioeconômicas são desfavoráveis.

\section{Colaboradores}

EV Müller, MGH Biazevic, JLF Antunes e EM Crosato participaram igualmente de todas as etapas de elaboração do artigo. 


\section{Referências}

1. Moore DH. Cervical cancer. Obstet Gynecol. 2006; 107:1152-1161.

2. Parkin DM, Bray FI, Devesa SS. Cancer burden in the year 2000: the global picture. Eur J Cancer. 2001; 37(Suppl.8):S4-S66.

3. Internacional Agency for Research on Cancer (IARC). Cervical cancer. [periódico na Internet]. [acessado 2005 jun 4]. Disponível em: http:// screening.Iarc.fr/cervicalindex

4. Stewart BW, Kleihues P. World cancer report. In: Stewart BW, Kleihues P, editors. Cancers for the female reproductive tract. Lyon: IARC Press; 2003. p. 215-218.

5. Behtash N, Mehrdad N. Cervical cancer: screening and prevention. Asian Pac J Cancer Prev 2006; 7:683686.

6. Shanta V, Krishnamurthi S, Gajalakshmi CK, Swaminathan R, Ravichandran K. Epidemiology of cancer of the cervix: global and national perspective. $J$ Indian Med Assoc 2000; 98:49-52.

7. Parikh S, Brennan P, Boffetta P. Meta-analysis of social inequality and the risk of cervical cancer. Int J Cancer 2003; 105:687-691.

8. Fonseca LAM, Ramacciotti AS, Neto JE. Tendência da mortalidade por câncer do útero no município de São Paulo entre 1980 e 1999. Cad Saude Publica 2004; 20:136-142.

9. Derossi SA, Paim JS, Aquino E, Silva LMV. Evolução da mortalidade e anos potenciais de vida perdidos por câncer cérvico uterino em Salvador (BA), 1979-1997. Rev Bras Cancerologia 2001; 47:163-170.

10. Kalakun L, Bozzetti MC. Evolution of uterine cervical cancer mortality from 1979 to 1998 in the State of Rio Grande do Sul, Brazil. Cad Saude Publica 2005; 21:299-309.

11. Nieminen P, Kotaniemi L, Hakama M, Tarkkanen J, Martikainen J, Toivonen T, Ikkala J, Luostarinen T, Anttila A. A randomised public-health trial on automation-assisted screening for cervical cancer in Finland: performance with 470,000 invitations. Int $J$ Cancer 2005; 115:307-311.

12. Guarisi R, Hardy E, Derchain SFM, Fonsechi-Carvasan GA, Borges JBR. Rastreamento diagnóstico e tratamento das lesões precursoras e do câncer invasor de colo uterino no município de Franco da Rocha, SP. Rev Bras de Cancerologia 2004; 50:7-15.

13. Brasil. Ministério da Saúde. Instituto Nacional de Câncer. Programa Nacional de Controle do Câncer do Colo Uterino: Viva Mulher. Rio de Janeiro: Ministério da Saúde; 1996.

14. Brasil. Ministério da Saúde. Datasus. Sistema de Informação de Mortalidade: 1979-2000 .[documento na Internet]. Brasília: Ministério da Saúde; 2000. [acessado 2005 jun. 4]. Disponível em: http:// www.datasus.gov.br/mortalidade

15. Organização Mundial da Saúde (OMS). Classificação internacional das doenças, $9^{a}$ revisão (CID-9). São Paulo: EdUSP; 1993.

16. Organização Mundial da Saúde (OMS). Classificação internacional das doenças e problemas relacionados à saúde, 10a revisão (CID-10). São Paulo: EdUSP; 1999.
17. Paraná. Secretaria de Estado da Saúde do Paraná (SESA). Mortalidade por câncer de colo de útero no Estado do Paraná. [acessado 2005 jun. 5]. Disponível em: http://saúde.pr.gov.br/cancerestimativa.htm

18. Brasil. Ministério do Planejamento, Orçamento e Gestão. Instituto Brasileiro de Geografia e Estatística (IBGE). População residente - Censo 2000: Brasil, unidades da federação e municípios, 2000. Rio de Janeiro: IBGE; 2000. [base de dados na Internet]. [acessado 2005 jun. 5]. Disponível em: http://www. ibge.gov.br

19. Pereira MG. Epidemiologia: teoria e prática. Rio de Janeiro, Guanabara; 2003.

20. Gaynor PE, Kirkpatrick RC. Introduction to timeseries modeling and forecasting in business and economics. New York: McGraw-Hill; 1994

21. Schiffman MH, Brinton LA, Devesa SS, Fraumeni JF Jr. Cervical cancer. In: Schottenfeld D, Fraumeni JF Jr, editors. Cancer epidemiology and prevention. $2^{\text {nd }}$ ed. New York: Oxford University Press; 1996. p. 1090-1116.

22. Franco EL, Duarte Franco E, Ferenczy A. Cervical cancer: epidemiology, prevention and the role of human papillomavirus infection. CMAJ 2001; 165(6):739-740.

23. Fonseca LA, Ramacciotti AS, Eluf Neto J. Mortality trends from uterine cervical cancer in the city of São Paulo from 1980 to 1999.Cad Saude Publica 2004; 20:136-142.

24. Prado MCO. Câncer feminino: evolução da mortalidade por câncer de mama e do colo do útero em distritos do município de São Paulo, 1985-1999 [tese]. São Paulo: Faculdade de Saúde Pública da Universidade de São Paulo; 2002.

25. Paes NA, Albuquerque MEG. Avaliação dos dados populacionais e cobertura dos registros de óbitos para as regiões brasileiras. Rev Saude Publica 1999; 33:33-43.

26. Wünsch Filho V, Moncau JE. Mortalidade por câncer no Brasil 1980-1995: padrões regionais e tendências temporais. Rev Assoc Med Bras 2002; 48:250-257.

27. Coker AL, Du XL, Fang S, Eggleston KS. Socioeconomic status and cervical cancer survival among older women: findings from the SEER - Medicare linked data cohorts. Gynecol Oncol 2006; 102:278-284.

28. Robles SC, White F, Peruga A. Trends in cervical cancer mortality in the Americas. Bull Pan Am Health Organ 1996; 30:290-301.

29. World Health Organization (WHO). Cytological screening in the control of cervical cancer: technical guidelines. Genebra: WHO; 1988.

30. Mendonça GAS. Câncer na população feminina brasileira. Rev Saude Publica 1993; 27:68-75.

31. Antunes JLF, Wünsch Filho V. The effect of performing corrections on reported uterine cancer mortality data in the city of São Paulo. Brazilian Journal of Medical and Biological Research 2006; 39:1091-1099.

Artigo apresentado em 19/04/2009

Aprovado em 28/08/2009

Versão final apresentada em 20/09/2009 